\title{
Comparison of Intranasal Outer Membrane Vesicles with Cholera Toxin and Injected MF59C.1 as Adjuvants for Malaria Transmission Blocking Antigens AnAPN1 and Pfs48/45
}

\author{
Michael Pritsch, ${ }^{1,2,3}$ Najib Ben-Khaled, ${ }^{1}$ Michael Chaloupka, ${ }^{4,5}$ Sebastian Kobold, ${ }^{4,5}$ \\ Nicole Berens-Riha, ${ }^{1}$ Annabell Peter, ${ }^{2}$ Gabriele Liegl, ${ }^{2}$ \\ Sören Schubert, ${ }^{2}$ Michael Hoelscher, ${ }^{1,3}$ Thomas Löscher, ${ }^{1}$ and Andreas Wieser ${ }^{1,2,3,6}$ \\ ${ }^{1}$ Division of Infectious Diseases and Tropical Medicine, Medical Center of the University of Munich (LMU), 80802 Munich, Germany \\ ${ }^{2}$ Department of Bacteriology, Max von Pettenkofer Institute (LMU), 81337 Munich, Germany \\ ${ }^{3}$ German Center for Infection Research (DZIF), Partner Site Munich, 80802 Munich, Germany \\ ${ }^{4}$ Center of Integrated Protein Science Munich (CIPS-M) and Division of Clinical Pharmacology, Department of Internal Medicine IV, \\ University of Munich (LMU), 80337 Munich, Germany \\ ${ }^{5}$ German Center for Lung Research, Munich, Germany \\ ${ }^{6}$ College of Public Health and Medical Science, Jimma University, Jimma, Ethiopia
}

Correspondence should be addressed to Michael Pritsch; pritsch@lrz.uni-muenchen.de

Received 13 December 2015; Revised 23 March 2016; Accepted 23 March 2016

Academic Editor: Kurt Blaser

Copyright (C) 2016 Michael Pritsch et al. This is an open access article distributed under the Creative Commons Attribution License, which permits unrestricted use, distribution, and reproduction in any medium, provided the original work is properly cited.

Purified protein vaccines often require adjuvants for efficient stimulation of immune responses. There is no licensed mucosal adjuvant on the market to adequately boost the immune response to purified antigens for intranasal applications in humans. Bacterial outer membrane vesicles (OMV) are attractive candidates potentially combining antigenic and adjuvant properties in one substance. To more precisely characterize the potential of Escherichia coli OMV for intranasal vaccination with heterologous antigens, immune responses for AnAPN1 and Pfs48/45 as well as ovalbumin as a reference antigen were assessed in mice. The intranasal adjuvant cholera toxin (CT) and parenteral adjuvant MF59C.1 were used in comparison. Vaccinations were administered intranasally or subcutaneously. Antibodies (total IgG and IgM as well as subclasses IgG1, IgG2a, IgG2b, and IgG3) were measured by ELISA. T cell responses (cytotoxic T cells, Thl, Th17, and regulatory T cells) were determined by flow cytometry. When OMV were used as adjuvant for intranasal immunization, antibody and cellular responses against all three antigens could be induced, comparable to cholera toxin and MF59C.1. Antigen-specific IgG titres above $1: 10^{5}$ could be detected in all groups. This study provides the rationale for further development of OMV as a vaccination strategy in malaria and other diseases.

\section{Introduction}

Vaccines are one of the most cost-effective measures in the field of public health and greatly reduce disease, disability, death, and inequity worldwide $[1,2]$. Their pivotal role has been demonstrated in infectious disease elimination campaigns (e.g., against smallpox, polio, or measles). However, due to the diversity of pathogens and their specific requirements for immune elimination or prevention, vaccination strategies cannot be readily translated from one disease to another, but they rely on a fine definition of protection and a good understanding of the immunological mechanisms underlying each vaccination [3].

Most vaccine formulations require adjuvant substances to boost immune responses, which have to be chosen according to their ability to induce the desired type of immune response without causing disproportional toxicity [4]. Another important consideration for vaccines is their application route. The most commonly used parenteral injection depends on the presence of sterile needles and medical personnel, which are 
major issues in parts of the world where infectious diseases are most prevalent. Mucosal vaccination strategies have the potential to overcome these limitations and are thereby in focus of vaccine research [5]. However, not every potential application route for mucosal vaccination may be socially and culturally acceptable. Oral or respiratory mucosal immunization strategies may have the highest acceptance and may circumvent many of the shortcomings of parenteral injections. Nonreplicating particles or recombinant proteins in combination with mucosal adjuvants can evoke mucosal and systemic immune responses [5]. Immune responses to vaccines also differ significantly in their ability to induce reactive $\mathrm{T}$ cells. Mucosal-especially intranasal (i.n.) - vaccines have been shown to produce strong $\mathrm{T}$ cellular responses [5]. These exquisite properties of mucosal vaccines are in sharp contrast to their current use, as only few vaccines have been approved so far for this indication (e.g., against polio, typhoid fever, or uropathogenic E. coli delivered orally or flu delivered as nasal spray); all of these consist of attenuated or inactivated pathogens. A major reason for this discrepancy is the lack of proper adjuvant substances [6].

Bacterial outer membrane vesicles (OMV) are particles about 100 nanometres in diameter produced by gramnegative organisms during growth. OMV encompass gramnegative outer membrane including transmembrane proteins as well as periplasmic matter. OMV are inherently potent immune-stimulators and are able to penetrate mucosal membranes as potent danger signal for innate immunity. Together with heterologous antigens, they can be used as adjuvant substances to promote immunity to these antigens [7-9]. Several studies have investigated the properties of Neisseria spp. OMV as vaccines as well as adjuvants [10-12]. It is currently unresolved how OMV would compare with conventional adjuvants in their capacity to induce immune reactions against malaria vaccine candidates.

Malaria has a huge impact on public health worldwide, causing 700,000 [13] to 1.2 million [14] deaths annually. Thus, there is a need to develop efficient strategies, such as transmission blocking vaccines. Among several others, two malaria antigens are deemed suitable for transmission blockade: (i) the Anopheles alanyl aminopeptidase N (AnAPN1) of the midgut of Anopheles mosquitoes has been found to play a critical role in Plasmodium falciparum and Plasmodium vivax ookinete invasion. Immunization against AnAPN1 was shown to inhibit Plasmodium replication in mosquitoes and with this stop transmission [15-18]; (ii) in the malaria parasite sexual stage, prefertilization gametocyte antigen Pfs48/45 was described to play a critical role in male gamete fertility, and vaccination can induce potent malaria transmission blocking antibodies in mice and nonhuman primates [19-27].

To characterize E. coli OMV as mucosal vaccine adjuvant in comparison to established adjuvants, mice were vaccinated with either AnAPN1, Pfs48/45, or ovalbumin (OVA) using intranasal OMV, intranasal cholera toxin (CT), or subcutaneous MF59C.1, respectively. Humoral and cellular responses were measured after 31 days. This study demonstrates that OMV elicit robust humoral and cellular immune responses against the tested antigens. Antibody titres were found to be comparable between the vaccination groups using the three different adjuvants. This study provides evidence for further evaluation of OMV for vaccination against malaria and other infectious diseases.

\section{Materials and Methods}

2.1. Construction, Expression, and Purification of Proteins. The amino acid sequences of AnAPN1 and Pfs48/45 were used as target antigens for the study. In case of AnAPN1, a 135amino-acid fragment containing the residues 61-194 located downstream of the N-terminus of mature AnAPN1 was used as to preserve also the C-terminal transmission blocking peptide 9 , as described previously $[15,17]$. In case of Pfs $48 / 45$, the native sequence (accession number AF356146) lacking the N-terminal signal sequence (amino acid residues 1-27) and the C-terminal anchor (amino acid residues 428-448) was used as described before [26]. Amino acid sequences were reversely translated into coding DNA sequences taking into account the optimized codon usage for Enterobacteriaceae as well as RNA secondary structures and other prominent sequences such as chi sites (DNAStar Inc., Madison, WI, USA). The resulting DNA sequences were synthetically generated by GeneArt (Regensburg, Germany). Synthetic genes were produced including flanking restriction sites for simplified cloning. For purification, the well-characterized 6His tag was fused to the N-terminus of each vaccine protein. The gene constructs were subcloned into pASK-iba37+ Plasmids (IBA-Lifesciences, Goettingen, Germany), using the restriction sites KpnI/PstI for AnAPN1 and KpnI/HindIII for Pfs48/45, respectively. Final plasmids were electroporated into the E. coli strain Top-10 and induced with anhydrotetracycline (AHT). Induction was performed as described by the manufacturer of the plasmid (IBA-Lifesciences, Goettingen, Germany). In brief, the specific strain was grown in LB containing ampicillin at a final concentration of $100 \mu \mathrm{g} / \mathrm{mL}$ at $37^{\circ} \mathrm{C}$ under agitation. After an $\mathrm{OD}_{600}$ of 0.5 was reached, $\mathrm{AHT}$ was added to a final concentration of $0.2 \mu \mathrm{g} / \mathrm{mL}$. The induction was performed for $3 \mathrm{~h}$ at $37^{\circ} \mathrm{C}$ under agitation. Bacterial cell pellets were harvested by centrifugation of the induced culture at $5000 \times \mathrm{g}$ at $4^{\circ} \mathrm{C}$ for $20 \mathrm{~min}$ and subsequently lysed in lysis buffer. The proteins AnAPN1 and Pfs48/45, which were tagged with a 6-His tag at the N-terminal side, were purified using Ni-nitrilotriacetic acid (Ni-NTA) columns. After several wash steps, the proteins were gel filtered to ensure high purity. Elution was performed under denaturing conditions with buffer containing $5 \mathrm{mM}$ Imidazole and $7 \mathrm{M}$ urea.

Cell culture grade, pyrogen-free OVA was purchased from Sigma-Aldrich (St. Louis, MO, USA); control protein His-DHFR-m45 stock solution was obtained from previous productions [28].

2.2. SDS-PAGE and Immuno-Blot Analysis. Discontinuous one-dimensional sodium dodecyl sulfate-polyacrylamide gel electrophoresis (SDS-PAGE) was performed with a Protran II Mini-Vertical unit (Bio-Rad, Munich, Germany). After electrophoresis, gels were either stained with Coomassie Blue or transferred to a nitrocellulose membrane (Trans Blot Cell; Bio-Rad, Munich, Germany). The blot was blocked with 3\% $(\mathrm{w} / \mathrm{v})$ bovine serum albumin (BSA) in phosphate buffered 
saline (PBS) supplemented with $0.5 \%(\mathrm{v} / \mathrm{v})$ Tween and incubated with diluted mouse serum. Preimmune or postimmune sera were used, respectively, followed by goat anti-mouse immunoglobulin (A, M, or G) peroxidase conjugate (SigmaAldrich, Germany). Blots were developed using enhanced chemiluminescence (ECL) detection reagents (Amersham Pharmacia Biotech/GE, Freiburg, Germany). Serum dilutions for IgM were $1: 1,000$ and for IgG 1:20,000. Dilutions of secondary antibodies used for blot development were $1: 4,000$ for IgG and 1:2,000 for IgM.

\subsection{Production of Adjuvants and Quantification of OMV.} Cholera toxin (CT) was obtained from Quadratech Diagnostics Ltd. (Epsom, UK). MF59C.1 was produced by Marien-Apotheke München (Munich, Germany). OMVs were obtained by harvesting from the supernatant of growing bacterial cultures, as described previously [29]. In brief, E. coli strain AW OMV01 (Wieser at al., manuscript in preparation) bacteria were inoculated into prefiltered $\mathrm{LB}$ broth $(0.22 \mu \mathrm{m}$ pore size filters; Millipore/Merck, Darmstadt, Germany) and grown under vigorous agitation and aeration at $37^{\circ} \mathrm{C}$ to an $\mathrm{OD}_{600}$ up to 2.0 . Bacteria were removed from the culture by centrifugation. The supernatant was sterile filtered with $0.45 \mu \mathrm{m}$ pore size filter (Millipore/Merck, Darmstadt, Germany). The OMVs were enriched from filtrates by ultracentrifugation at $150,000 \times \mathrm{g}$ at $4^{\circ} \mathrm{C}$ for $3 \mathrm{~h}$. The resulting translucent pellet was suspended in sterile phosphate buffered saline (PBS) and stored at $4^{\circ} \mathrm{C}$ in the fridge until use for a maximum of 3 days. Quantification of OMV was performed as described before [29] using flow cytometry as well as Bradford protein measurements.

2.4. Vaccination of Animals. Animal experiments were carried out in strict accordance with the recommendations in the guidelines of the Federation of European Laboratory Animal Science Associations. On June 1st, 2012, the accountable German authority (Government of Upper Bavaria, Munich, Germany) approved the study protocol. Animals used for vaccinations were 6- to 8-week-old female pathogen-free BALB/c mice (Janvier, Saint-Berthevin, France), $14.9 \mathrm{~g}$ to $20.3 \mathrm{~g}$ of weight. All animals were housed under specific pathogen-free (SPF) conditions in individually ventilated positive pressure cabinets (Tecniplast, Hohenpeissenberg, Germany) with controlled temperature and humidity as well as strict $12 \mathrm{~h}$ day/night cycle. Bedding was autoclaved before use; sterile water and sniff-extrudated food were offered ad libitum. Each experiment was repeated at least twice with 5 mice in each group. I.n. vaccination was performed on days $0,3,5$, and 21 . For vaccination, mice were anesthetized with isoflurane $4 \%$ for a few seconds in the anaesthesiology chamber of a narcosis device (XGI-8 Gas Anesthesia System; Xenogen Corporation, Alameda, Ca, USA). Anaesthetized mice were handled inside the laminar flow hood and the vaccine was delivered into each nostril using $20 \mu \mathrm{L}$ Eppendorf GE-Loader tips (Eppendorf, Hamburg, Germany). To increase stiffness, they were trimmed with sterile scissors to a total length of about $30 \mathrm{~mm}$ before use. Each nostril of the mouse was individually probed and $5 \mu \mathrm{L}$ of the aqueous vaccination solution was administered. The vaccine contained either (i) $10 \mu \mathrm{g}$ of the respective vaccine protein (AnAPN1, Pfs 48/45, or OVA) plus CT (Quadratech, Surrey, United Kingdom) $(0.5 \mu \mathrm{g} / 10 \mu \mathrm{L})$ or (ii) $10 \mu \mathrm{g}$ of the respective vaccine protein (AnAPN1, Pfs $48 / 45$, or ovalbumin) plus $5 \mu \mathrm{L}$ OMVs or (iii) CT $(0.5 \mu \mathrm{g} / 10 \mu \mathrm{L})$ or (iv) $5 \mu \mathrm{L}$ OMVs. Each (i-iv) solution was prepared in PBS with adjusted $\mathrm{pH}$ of 7.4. All solutions were freshly prepared and mixed again directly prior to the application. As an application control PBS was used (v).

For parenteral application, the subcutaneous (s.c.) vaccination route was chosen inspired by the data of Valensi et al. [30]. In pilot experiments, we could detect robust immune responses using subcutaneous immunization rather than intramuscular while maintaining better tolerability on the side of the laboratory animal. Doses were administered on days 0,7 , and 21; a total amount of $20 \mu \mathrm{L}$ of an aqueous solution was inoculated into the scruff of the neck, containing either (i) $10 \mu \mathrm{g}$ of the respective vaccine protein (AnAPN1, Pfs48/45, or OVA) plus $10 \mu \mathrm{g}$ MF59C.1 or (ii) $10 \mu \mathrm{g}$ MF59C.1, each (i, ii) diluted in PBS, or (iii) PBS only as application control.

All mice were sacrificed on day 31 to obtain mouse sera and splenocytes to investigate the evoked immune responses. Throughout the experiments, mice were monitored daily for abnormalities of behaviour. The weight was determined on a weekly basis.

2.5. Measurement of the Immune Responses in the Mouse. The antibody response as well as the $\mathrm{T}$ cellular response to the vaccinations was analysed with different methods.

(I) Humoral Immune Response. Serum antibody titres were determined with ELISA. Titres of total IgG and IgM as well as the subclasses IgG1, IgG2a, IgG2b, and IgG3 were determined for each respective antigen and administration route. For specific serum antibody detection, Nunc Immuno Plates (Maxisorp F96 flat bottom plate, Sigma-Aldrich, Wiesbaden, Germany) were coated with the respective antigen by incubation of $100 \mu \mathrm{L}$ of antigen at $20 \mu \mathrm{mol} / \mathrm{mL}$ in carbonate-bicarbonate buffer at $4^{\circ} \mathrm{C}$ overnight. The plate was subsequently washed twice with PBS containing 0.05\% Tween 20 (PBS-T). Nonspecific bindings were saturated with $200 \mu \mathrm{L}$ of blocking buffer ( $1 \%$ bovine serum albumin in PBS with $0.05 \%$ Tween 20 ) for $1 \mathrm{~h}$ at $37^{\circ} \mathrm{C}$. Serum samples were serially diluted in blocking solution, starting with a 100 -fold dilution, and measured in triplicate (some individual mouse sera were measured in duplicate because of small sample volume). Endpoint dilution data from a series of 16 steps between $1: 10^{2}$ and $1: 10^{6}$ are presented in Figures 1-3. A $100 \mu \mathrm{L}$ of diluted serum was incubated for $1 \mathrm{~h}$ at $37^{\circ} \mathrm{C}$, followed by four PBS-T washing steps. After addition of the secondary antibodies, plates were incubated for $1 \mathrm{~h}$ at $37^{\circ} \mathrm{C}$ and subsequently washed again four times with PBS-T. Secondary antibodies conjugated with horseradish peroxidase (HRP) were used for detection. Read-out was performed with o-phenylenediamine substrate (Sigma-Aldrich, Wiesbaden, Germany) in $0.1 \mathrm{M}$ phosphate/citrate buffer ( $\mathrm{pH}$ 5.0). The substrate was incubated for $30 \mathrm{~min}$ at room temperature $\left(21 \pm 1^{\circ} \mathrm{C}\right)$ under light protected conditions. 


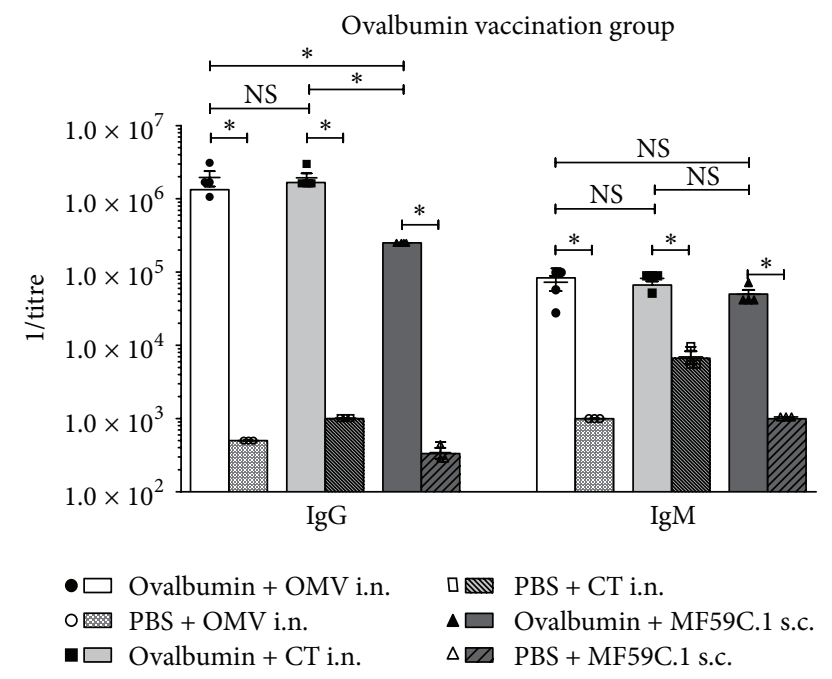

(a)

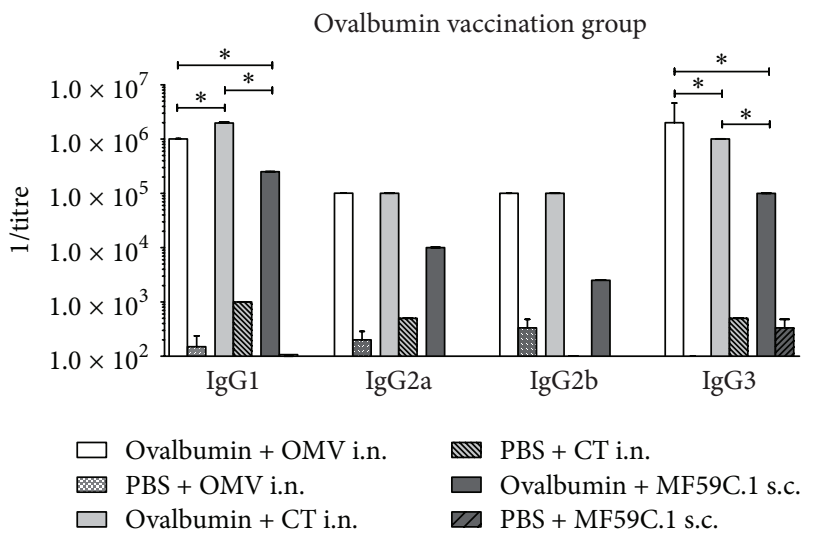

(b)

FIgURE 1: Characterization of the humoral response to ovalbumin vaccination. Each column depicts a representative vaccination group consisting of five mice, respectively. (a) depicts whole IgG and IgM titres in direct comparison between the three adjuvants. Single mouse as well as mixed mouse serum data is shown including standard deviations. For control groups, only three individual mouse sera were tested additionally to the mixed serum. (b) shows IgG1, $-2 a,-2 b$, and -3 titres of mixed mouse sera in comparison for the three adjuvant substances including controls. Titres of $<10^{2}$ have not been determined and thus are not depicted. NS = nonsignificant; $*=$ significant difference $p<0.05$; OMVs $=$ bacterial outer membrane vesicles; $\mathrm{CT}=$ cholera toxin; i.n. $=$ intranasal vaccination; s.c. $=$ subcutaneous vaccination.

Absorbance was measured with a plate reader (Tecan, Grödig, Austria) at a wavelength of $492 \mathrm{~nm}$ according to the instructions of the manufacturer. Secondary antibodies were chosen depending on the desired measurement. Whole IgG titres were determined using goat anti-mouse IgG (Sigma-Aldrich, Wiesbaden, Germany) diluted 1:1,000 in blocking solution; anti-IgM specific and anti-IgG subclass specific antibodies (goat anti-mouse IgM, IgG1, IgG2a, IgG2b, and IgG3) were purchased at Santa Cruz Biotechnology (Santa Cruz, CA, USA) and used in a dilution of 1:3,000 in blocking buffer.

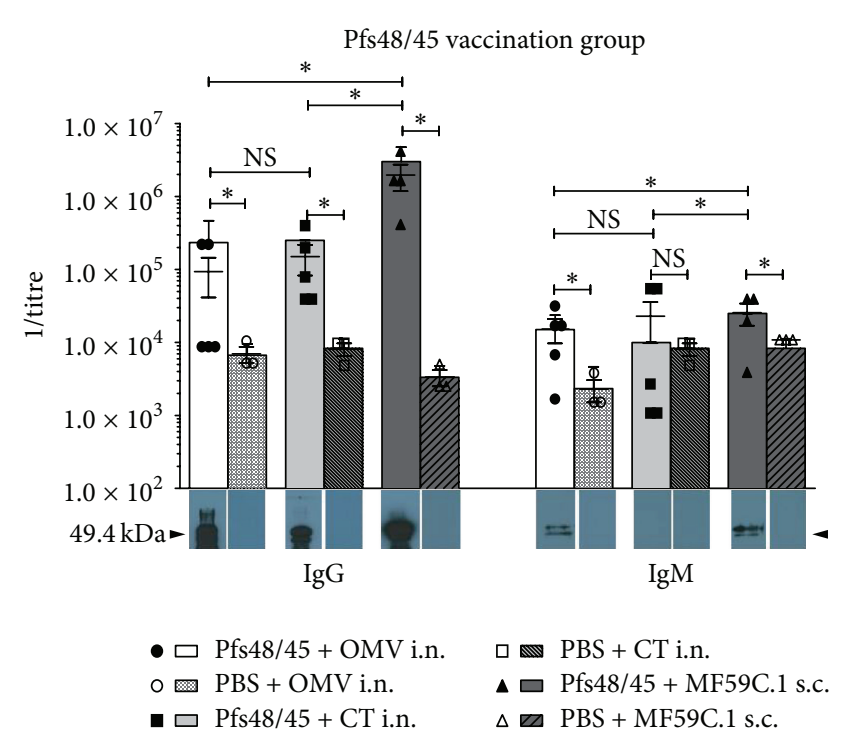

(a)

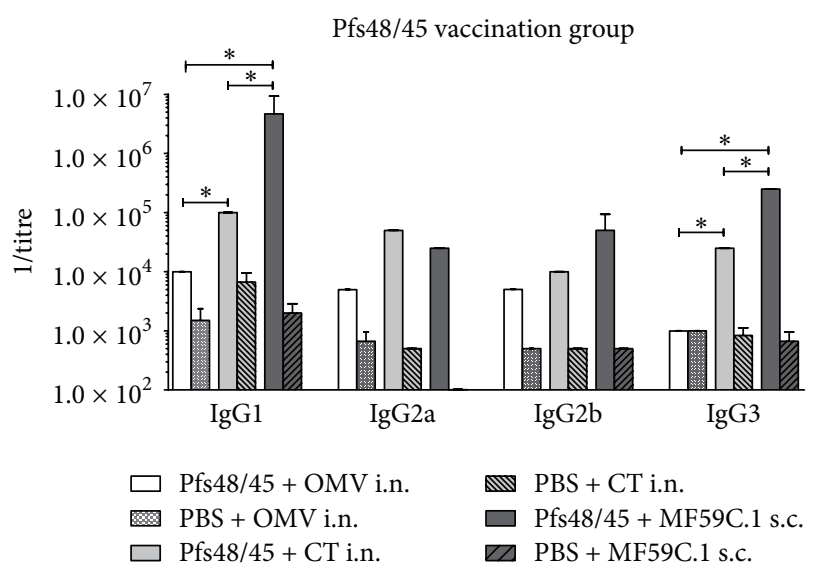

(b)

Figure 2: Characterization of the humoral response to Pfs $48 / 45$ vaccination. Each column depicts a representative vaccination group consisting of five mice, respectively. (a) depicts whole IgG and IgM titres in direct comparison between the three adjuvants. Single mouse as well as mixed mouse serum data is shown including standard deviations. For control groups, only three individual mouse sera were tested additionally to the mixed serum. Immuno-Blot analysis was performed as a second line of evidence. (b) shows IgG1, $-2 a,-2 b$, and -3 titres of mixed mouse sera in comparison for the three adjuvant substances including controls. Titres of $<10^{2}$ have not been determined and thus are not depicted. $\mathrm{NS}=$ nonsignificant; $*=$ significant difference $p<0.05$; OMVs = bacterial outer membrane vesicles; $\mathrm{CT}=$ cholera toxin; i.n. $=$ intranasal vaccination; s.c. $=$ subcutaneous vaccination.

Statistical analysis was performed with the mean optical density (OD) of each triplicate. A titre measurement was considered positive when the mean $\mathrm{OD}$ value was greater than the mean $\mathrm{OD}$ of the negative controls plus 10 standard deviations (SD). 


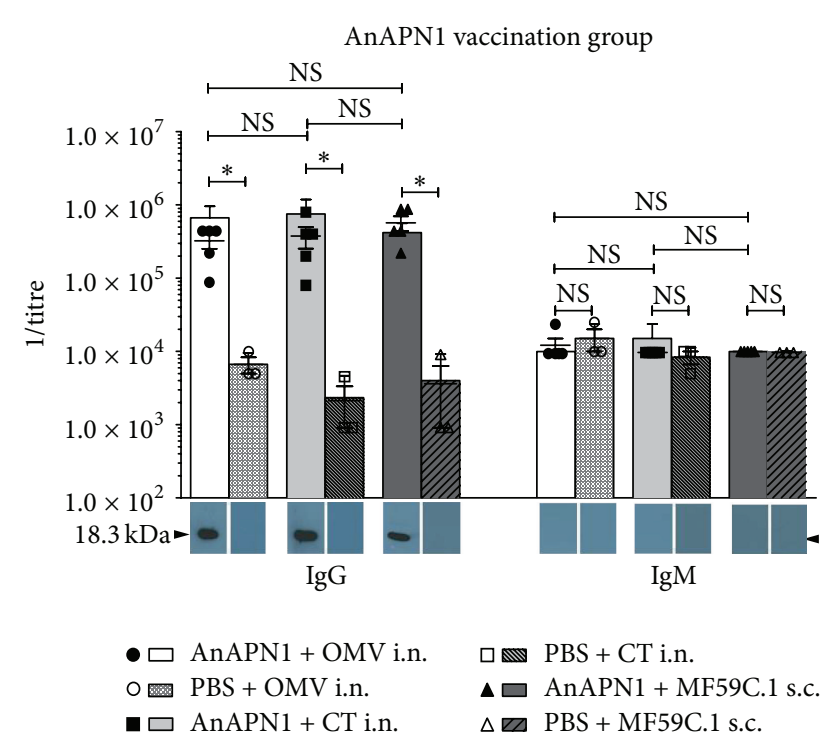

(a)

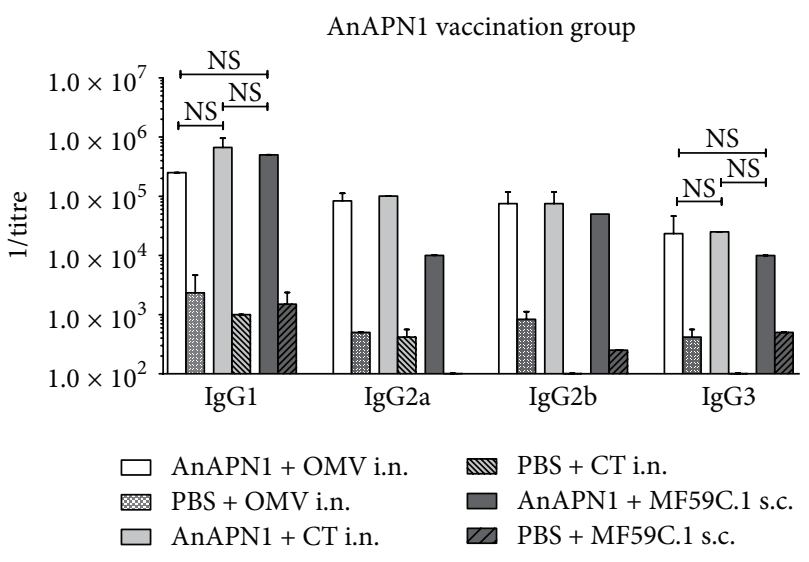

(b)

FIGURE 3: Characterization of the humoral response to AnAPN1 vaccination. Each column depicts a representative vaccination group consisting of five mice, respectively. (a) depicts whole IgG and IgM titres in direct comparison between the three adjuvants. Single mouse as well as mixed mouse serum data is shown including standard deviations. For control groups, only three individual mouse sera were tested additionally to the mixed serum. Immuno-Blot analysis was performed as a second line of evidence. (b) shows IgG1, $-2 a,-2 b$, and -3 titres of mixed mouse sera in comparison for the three adjuvant substances including controls. Titres of $<10^{2}$ have not been determined and thus are not depicted. NS = nonsignificant; $*=$ significant difference $p<0.05$; OMVs $=$ bacterial outer membrane vesicles; $\mathrm{CT}=$ cholera toxin; i.n. $=$ intranasal vaccination; s.c. $=$ subcutaneous vaccination.

(II) Cellular Immune Response. After completion of the vaccination experiment, mice were sacrificed and spleens were aseptically removed. Spleens were lysed in lysis buffer (Passive Lysis Buffer, Promega, Madison, MI, USA) and homogenized in RPMI 1640 with $10 \%$ FCS, $1 \%$ Pen/Strep, and $1 \%$ LGlutamine at a concentration of $7.5 \times 10^{6} / \mathrm{mL}$ per well in $200 \mu \mathrm{L}$. Cell count was determined with a Neubauer-chamber.
Single spleen cell suspensions of the vaccinated mice were pulsed over night with the relevant proteins at the indicated concentrations (AnAPN1, 0.1 mg/mL; Pfs $48 / 45,0.1 \mathrm{mg} / \mathrm{mL}$; OVA, $0.5 \mathrm{mg} / \mathrm{mL}$; or the equivalent volume of PBS). Cells were restimulated with PMA $(50 \mathrm{ng} / \mathrm{mL}$, Sigma-Aldrich, Wiesbaden, Germany) and Ionomycin $(1 \mu \mathrm{g} / \mathrm{mL}$, SigmaAldrich) for $5 \mathrm{~h}$ with the addition of Brefeldin A $(1 \mu \mathrm{g} / \mathrm{mL}$, Sigma-Aldrich) after $1 \mathrm{~h}$. Cells were surface stained with anti-CD3-Pe-Cy7 (clone 145-2C11, Biolegend, San Diego, CA, USA), anti-CD4-PerCP (clone GK1.5, Biolegend), and antiCD8-APC-Cy7 (clone 53-6.7, Biolegend). Cells were fixed and permeabilised using a Foxp3/Transcription Factor Staining Buffer Set (eBioscience, San Diego, CA, USA). Intracellular staining was performed with anti-IFN- $\gamma$-PE (clone XMG1.2, Biolegend), anti-IL-17-FITC (clone TC11-18H10.1, Biolegend), and anti-Foxp3-Pacific Blue (clone FJK-16s, eBioscience). Cells were analysed using a BD FACS Canto II (BD Biosciences, Franklin Lakes, NJ, USA).

2.6. Statistical Analyses. Individual data sets were analysed using the SigmaStat Software package (Systat Software $\mathrm{GmbH}$, Erkrath, Germany). ELISA data were compared with the Mann-Whitney rank sum test, as data was not always normally distributed; flow cytometric data was analysed using Student's $t$-test. $p$ values of $<0.05$ were considered statistically significant and marked with $*$.

\section{Results}

3.1. Preparation of AnAPN1, Pfs $48 / 45$, and $O M V$. The G/C contents of AnAPN1 and Pfs $48 / 45$ sequence after codon harmonization were $49.19 \%$ and $41.63 \%$, respectively (see Text S1 in the Supplementary Material available online at http://dx .doi.org/10.1155/2016/3576028). Protein properties can be found in more detail in the Supplementary Material (Text S2). Codon usage was significantly improved for enterobacterial preference in the synthetic genes. Expression and purification were performed as described in more detail in Section 2 (see also Text S3). Induction in transgenic E. coli yielded sufficient protein amounts for vaccination experiments; the concentration of vaccine protein in the final elution sample was $4.5 \mathrm{mg} / \mathrm{mL}$ for AnAPN1 and $3.2 \mathrm{mg} / \mathrm{mL}$ for Pfs $48 / 45$, respectively. The lipopolysaccharide content of the protein preparations was determined and found to be equal to an LPS load for each administration $(10 \mu \mathrm{g})$ of $0.02 \mathrm{ng}$ in the case of AnAPN1 and $0.03 \mathrm{ng}$ for Pfs $48 / 45$, respectively. OMVs were produced as described above and before [29]. Emulsified OMVs were diluted to a concentration of $5 \times 10^{6} / \mu \mathrm{L}$ for the use as adjuvant.

3.2. Immunization. In each vaccination group, a total of five mice were vaccinated. Individual experiments were repeated twice independently. Vaccinations were well tolerated without any noticeable local or systemic adverse events. Laboratory animals were observed daily and with special scrutiny the hours following the administration of the vaccines. Standardized score sheets were used to document behaviour and weight. No significant difference in weight gain or behaviour could be observed between groups; no signs of acute toxicity could be observed. 
3.3. ELISA. In the OVA groups, total IgG titres reach up to $1: 10^{6}$, equally distributed between the i.n. vaccination routes (Figure 1). The titres of s.c. vaccination with MF59C.1 were significantly lower than with both i.n. routes. For all three vaccines, IgG1 is the main IgG subclass evoked, again with the intranasal routes being superior to the s.c. administration. IgG3 subclass titres were found at similar levels compared to IgG1 for all vaccine routes. IgM titres are only roughly one order of magnitude below IgG titres even after one month. All titres increased significantly during the course of vaccination. Detectable IgM persisted throughout the experiment in the groups of all three adjuvants. Although the overall IgG titres in the MF59C.1 group were lower, the IgM titres were not significantly different in all three groups.

In the groups vaccinated with Pfs $48 / 45$, total IgG titres were in the range of $>10^{5}-10^{6}$ (Figure 2).

In Western Blot analysis, a strong IgG reaction can be detected. Interestingly, a higher molecular mass band can be seen in the OMV i.n. Pfs $48 / 45$ group and to a lesser extent in the other Pfs $48 / 45$ vaccinated groups. It could not be detected in the mock-immunized groups. Mice vaccinated with i.n. OMV and to a lesser extent those vaccinated with MF59C.1 had persistent IgM and low IgG3 titres. However, substantial IgM titres were observed in the ELISA measurements also of the mock-immunized groups. Thereby, the Western Blot analysis could only detect a specific reaction in the Pfs $48 / 45$ groups immunized with OMV as well as MF59C.1, which also demonstrated a significantly higher titre in the vaccinated group as compared to the mock-immunized control (Figure 2). In contrast, mice vaccinated with i.n. CT had no persistent IgM but had higher IgG3, with titres for IgG3 being higher than those for IgG2b. Most of the IgG response was of the IgG1 subclass in all vaccination groups. OMV vaccinated mice had, however, lower IgG1 titres compared to the other groups. Overall, titres achieved with the s.c. vaccination were significantly higher than in the other two groups.

Mice vaccinated with AnAPN1 developed high titres of up to $10^{6}$ (Figure 3). Mucosal vaccination showed a trend towards higher titres compared to the parenteral vaccination. For all groups, no IgM persistence above the titres of the mock-immunized groups could be observed and IgG3 titres were lower than IgG2a and -b titres. MF59C.1 vaccination induced the lowest IgG2a titres.

When controlling all titres against a decoy protein (DHFR of the mouse) with identical N-terminal signal sequences and His tag as used in AnAPN1 and Pfs48/45, respectively, no deviation from background could be detected in any group, excluding unspecific binding to the purification tag sequences.

3.4. T Cell Response. OVA was used as model antigen, in order to analyse the ability of the used adjuvants (CT, MF59C.1, and OMV) to induce an antigen-specific cellular response. All three adjuvants were able to induce OVAspecific cytotoxic T cells (Figure 4(a)). CT was better than MF59C.1 and showed a trend towards stronger induction of cytotoxic T cells than OMV (Figure 4(a)). CT was the only adjuvant able to induce a Thl response towards OVA (Figure 4(b)), while MF59C.1 and OMV did not. None of the adjuvants induced a significant number of OVA-specific Th17 or regulatory $\mathrm{T}$ cells (Figures $4(\mathrm{c})$ and $4(\mathrm{~d})$ ).

In contrast, when comparing the ability of the said adjuvants to induce AnAPN1-specific cellular responses, only OMV was able to induce a cytotoxic $\mathrm{T}$ cell response towards AnAPN1, but all induced a Thl response towards the antigen (Figures 5(a) and 5(b)). Again, no induction of antigen-specific Th17 or regulatory T cells (Figures 5(c) and 5(d)) could be observed.

When assessing the responses induced by $\mathrm{Pfs} 48 / 45 \mathrm{vac}-$ cination, we found a decrease in cell number for all the conditions where cells have been restimulated with Pfs $48 / 45$ (Figure S4). All cell populations including cytotoxic T cells, Th1 cells, Th17 cells, and regulatory $\mathrm{T}$ cells decreased upon restimulation with Pfs48/45 (Figure S4).

\section{Discussion}

In the present study, humoral and cellular immune responses evoked by different vaccination methods using malaria transmission blocking antigens and ovalbumin in mice have been characterized and compared [15-27]. This study is the first to compare humoral and cellular immune responses for i.n. OMV adjuvating malaria transmission blocking antigens. Purified antigens from transgenic bacterial cultures (or purchased, in the case of OVA) were used and mixed with the respective adjuvant. This strategy does not make use of the full capacity of OMV for vaccination, as the antigens can be expressed directly inside the OMV [31]. To allow direct comparison of the vaccination routes, the same antigen batch was used with the different adjuvants. This approach considerably reduces possible artefacts due to folding abnormalities or contaminations of the used antigen preparation. However, this cannot control for antigen losses, which are to be expected due to uptake on the mucosal surface or sneezing out of the vaccination solution by the animals. To allow for a better evaluation of our results and comparison with literature, three previously studied antigens have been used. In this study, an adjuvant licensed for human use (MF59C.1) [32] and two mucosal adjuvants were used. While cholera toxin cannot be used in humans due to toxicity, it is considered highly potent and can be used as the reference substance in mice [33].

Previously, the use of modified OMV for i.n. vaccination has been reported and also been used as intranasal adjuvant [12], but concerns about their endotoxin (LPS) content is seen as problematic especially for parenteral application. To circumvent this issue, injectable formulations use extracted OMV without large amounts of LPS [34]. Mucosal surfaces may be less sensitive to LPS, since these are generally colonized by bacteria and thus potentially more amenable to safe OMV usage [35].

Humoral immune responses are key for the success of classic vaccine antigens [3]. For the malaria transmission blocking antigens AnAPN1 and Pfs48/45, antibodies are pivotal for transmission blockade in the midgut of the mosquitoes and IgGs seem to be of primary importance [1527]. Although, for malaria transmission blocking vaccines, the long-term antibody response is important and has not 


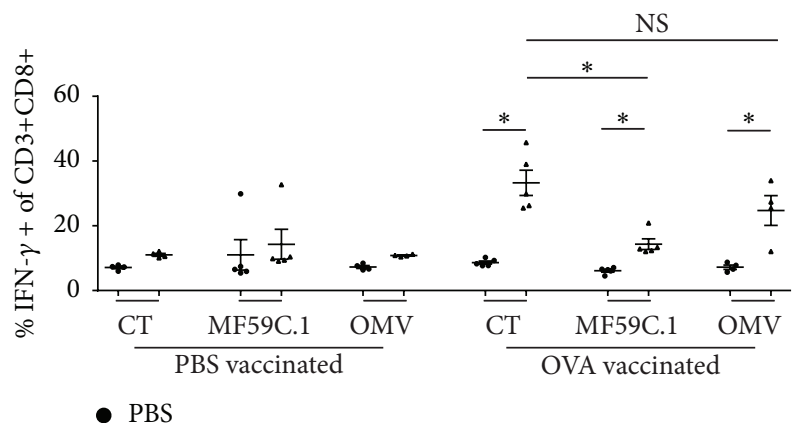

(a)

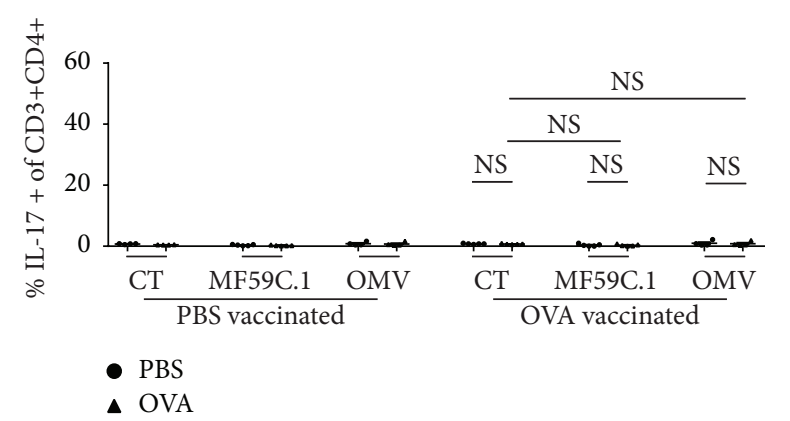

(c)

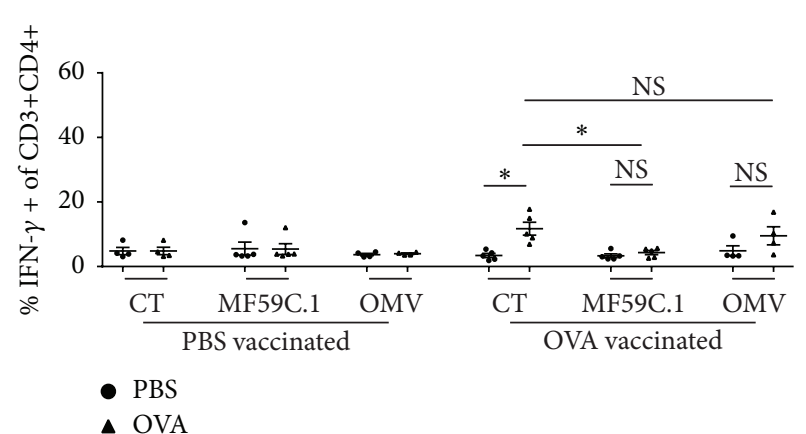

(b)

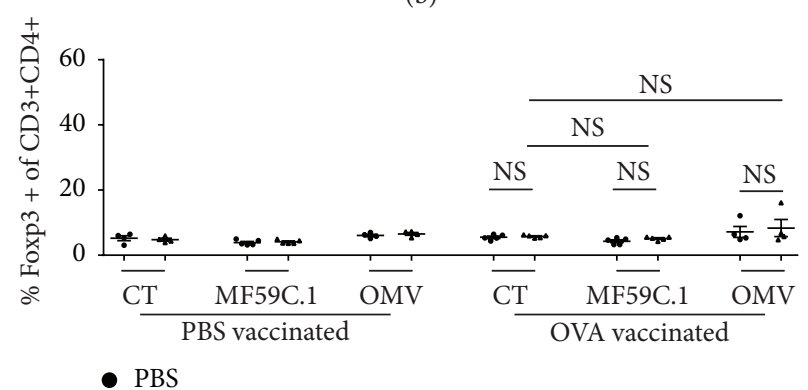

(d)

FIGURE 4: Characterization of the cellular response to ovalbumin vaccination. Each dot or triangle represents an individual mouse. Each PBS vaccination consisted of four mice and each ovalbumin vaccination group of five mice. Comparisons between groups were performed by Student's $t$-test. (a) Percentage of IFN-g secreting CD3 and CD8 double positive cells. (b) Percentage of IFN-g secreting CD3 and CD4 double positive cells. (c) Percentage of IL-17 secreting CD3 and CD4 double positive cells. (d) Percentage of Foxp3 positive CD3 and CD4 double positive cells. NS = nonsignificant; $*=$ significant difference $p<0.05$; OMVs $=$ bacterial outer membrane vesicles; CT $=$ cholera toxin.

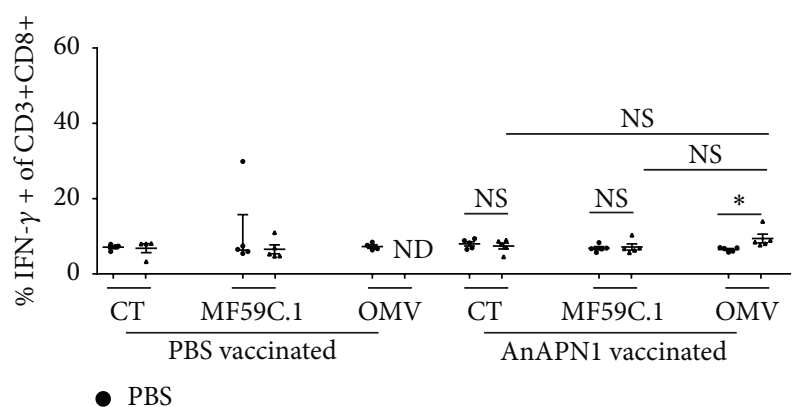

$\triangle$ AnAPN

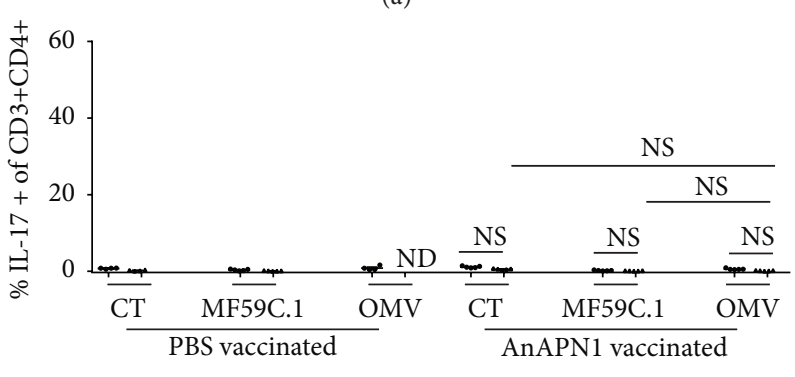

- PBS

$\triangle$ AnAPN1

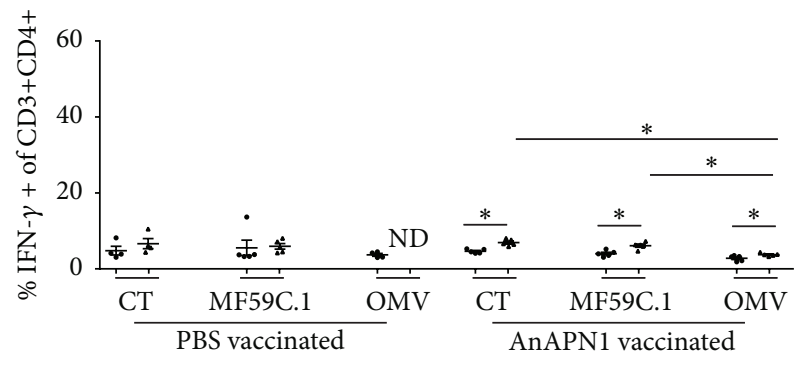

- PBS

- AnAPN1

(b)

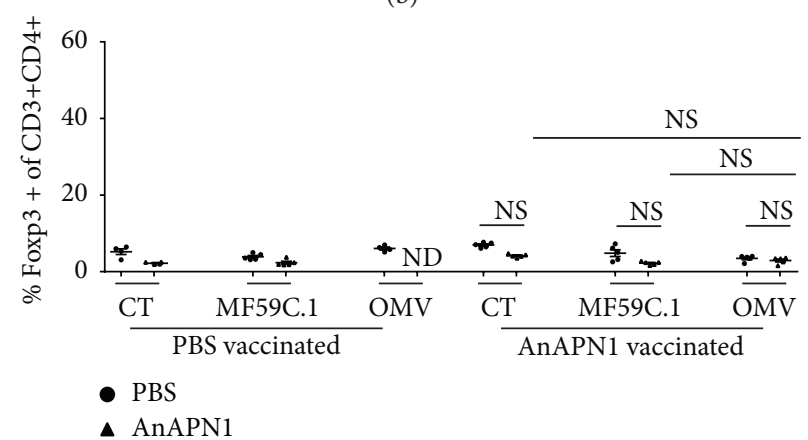

(d)

FIGURE 5: Characterization of the cellular response to AnAPN1 vaccination. Each dot or triangle represents an individual mouse. Each PBS vaccination consisted of four mice and each AnAPN1 vaccination group of five mice. Comparisons between groups were performed by Student's $t$-test. (a) Percentage of IFN-g secreting CD3 and CD8 double positive cells. (b) Percentage of IFN-g secreting CD3 and CD4 double positive cells. (c) Percentage of IL-17 secreting CD3 and CD4 double positive cells. (d) Percentage of Foxp3 positive CD3 and CD4 double positive cells. NS = nonsignificant; $*=$ significant difference $p<0.05 ;$ OMVs $=$ bacterial outer membrane vesicles; $\mathrm{CT}=$ cholera toxin; $\mathrm{ND}=$ not determined. 
been studied here, antibodies evoked at the peak of the immune response are of interest, offer valuable first insights into immunogenicity, and help with the design of studies covering the more antigen-specific application areas.

In the OVA vaccination groups, the total IgG titres of the i.n. groups were significantly higher than in the MF59C.1 adjuvated, s.c. administered animal batch (Figure 1). Injected OVA was previously found to be very immunogenic [36]. These high titres after i.n. vaccination are surprising, as OVA administered intranasally with liposomes was previously found to be less efficient than in combination with CT [37, 38]. High IgG3 titres, which were significantly lower than IgG1 in all groups, and persistent IgM were observed as well (Figure 1). This is noteworthy as two of the protocols involved the i.n. application of the antigen/adjuvants mixture rather than the injection. Therefore, it is possible that the properties of OVA as an antigen influenced the antibody subclass distribution rather than the application route or adjuvant substance. Other studies using different adjuvant substances reported IgG2a titres to be higher than IgG3 when injected with CpG, Bp, and CFA, respectively [36]. In these studies, however, i.n. routes have not been used at all and a clear influence of the adjuvant substance can be seen. OVA groups show a strong Th2 dependent response with IgG1 as the most dominant subclass (Figure 1). Nevertheless, IgG2a titres are only one order of magnitude lower in titre levels than IgG1 and are still in the range of $1: 10^{5}$ (Figure 1). This argues for a strong Th1 answer as well [39]. Besides these two, the $\mathrm{T}$ cell independent IgG3 reaches almost the same level as $\mathrm{IgG1}$, which is noteworthy.

Titres in the Pfs $48 / 45$ groups were on average similar to the OVA titres, however, with a tendency to be lower especially in the i.n. groups (Figure 2). The s.c. group vaccinated with MF59C.1 actually showed the highest titre measured in the experimental series described here. The titres for total IgG were not different between the i.n. groups, however, significantly higher in the s.c. group (Figure 2). In Western Blot analysis, a higher molecular weight band can be appreciated in the OMV i.n. Pfs $48 / 45$ vaccinated group and to much less extent also in the MF59C.1 and CT groups (see Figure 2(a)). This band is most likely a very slight reaction against traces of larger molecular weight protein found within the purified Pfs48/45 stock (see also silver stain in Supplementary Material (S3)), which is most prominently seen in the OMV vaccinated group. However, it is not present in the controls; thus a significant unspecific reaction can be excluded. Interestingly, IgM titres persisted significantly above the controls in the OMV i.n. group and the MF59C.1 group but not in the CT group (Figure 2). The OMV adjuvated i.n. administration also has low IgG3 levels as well as relatively low IgG2a levels (Figure 2). Therefore, the antibody response in this route seems to be mainly Th2 based. In contrast, the MF59C.1 groups showed significantly higher IgG3 titres (Figure 2). Thus, besides the strong Th2 mediated IgG1 response T cell independent IgG3 seems to be of greater importance than with the OMV adjuvated i.n. group. The CT i.n. group shows an intermediate phenotype as compared to the two other groups. Interestingly, the OMV adjuvant was able to stimulate a strong $\operatorname{IgG} 3$ response in the OVA-experiment and also strong Th1 mediated IgG2a titres in the OVA as well as AnAPN1 group (see Figures 1 and 3, resp.). IgG3 has been described before to be the second most important antibody subclass evoked after vaccination with Neisseria OMV [40]. The lack of IgG3 and IgG2a has to be attributed not to the properties of the adjuvant itself but rather to the combination of the adjuvant with the antigen, or possible penetration, or uptake problems of the antigen Pfs48/45 when administered in the i.n. application route. The overall size of the Pfs $48 / 45$ protein construct was relatively large with $49.4 \mathrm{kDa}$ and charge is comparatively strong $(-10.8$ at $\mathrm{pH} 7$ ), whereas AnAPN1 is much smaller and with less charge (18.3 $\mathrm{kDa}$ and -1.2 at $\mathrm{pH} 7)$, and OVA, although of roughly the same size $(45 \mathrm{kDa})$, is favourable regarding solubility and charge (Text S2). Still, the humoral immune response was strong in all groups, never reaching titres below $1: 10^{5}$ (Figures $1-3$ ).

Overall the OVA and Pfs45/48 vaccinations had a tendency of reaching higher average titre levels over all three vaccination routes (both $1.1 \times 10^{6}$ ) as compared to AnAPN1 $\left(6.1 \times 10^{5}\right)$ (Figure 3), arguing for all three application routes to be a relatively robust system, although the difference in size and physical properties are striking (Text S2). The AnAPN1 vaccination groups do not show significant differences between the application routes regarding the total IgG titres (Figure 3). There seems to be a trend towards higher titres on the side of the i.n. groups as compared to the s.c. group, but this was not significant. All groups lean towards an IgG1 based response, which is most dominant in the MF59C.1 group (Figure 3). Other than with OVA, the different subclass distribution patterns argue for a more Th1/2 balanced response with the OMV group and a mostly Th2 biased answer in the MF59C.1 group. All three groups show only relatively low IgG3 levels; thus T cell independent IgG3 might not be easily generated with AnAPN1, which is in accordance with a previous study using incomplete Freund's adjuvant and Alhydrogel in BALB/c and Swiss Webster mice [17]. This may be due to properties of the antigen itself rather than the adjuvant substance, as high IgG3 levels could be detected with all three adjuvants when applied together with OVA. Subclass antibody data published previously for Pfs $48 / 45$ vaccination trials also showed dominating IgG1 responses with highly variable contributions of $\operatorname{IgG} 2 \mathrm{a}, \operatorname{IgG} 2 \mathrm{~b}$, and $\operatorname{IgG} 3$ depending on adjuvants (Montanide ISA-51, Alum, CFA) and/or animal models (olive baboons, BALB/c mice) used [26].

The induction of $\mathrm{T}$ cell responses through vaccination does not only rely on the adjuvant and the administration route used, but also rely on the target antigen and its amenability to MHC-I or II presentation [41, 42]. All adjuvants of the present study were able to support cytotoxic $\mathrm{T}$ cell responses towards OVA (Figure 4(a)). However, only OMVs could induce such T cells against AnAPN1 (Figure 5(a)). Therefore, the difference observed with this antigen cannot be explained by the general capacity of the adjuvant to induce CD8 T cell responses, but rather by the nature of the antigen which may not be readily accessible to the MHC presenting machinery and requires adequate maturation and stimulation of antigen-presenting cells for proper immune induction as was described for other antigens [43]. A lack of proper 
presentation of AnAPN1 during restimulation in vitro can be ruled out, since reactive $\mathrm{T}$ cells could be detected in the case of OMV. In this setting, OMVs seem to mediate best antigen presentation of AnAPN1 in vivo leading to measurable immune responses compared to the other adjuvants. It is also important to note that no induction of antigenspecific regulatory $\mathrm{T}$ cells could be found which would have hampered the function of effector $\mathrm{T}$ cells and thus hijacked any therapeutic benefit [44]. CD8 positive $\mathrm{T}$ cells may be important for malaria protection but are not needed for malaria transmission blocking activity [15-27, 45]. However, levels can be used to better characterize the immune response supported by the individual route. The data, however, cannot preclude an advantage over other types of vaccination based on the induction of CD8 $\mathrm{T}$ cell responses [46].

While IgG1 was the most prominent antibody subtype detected for all antigens and adjuvants, only Th1 but not Th2 cellular responses could be detected upon restimulation. Th2 cells may be below the limit of detection. The analysis of antibody subtypes at the time point of sampling also revealed persistence of IgM, potentially indicating an incomplete class switch towards IgG at that time point. The analysis is, however, somewhat hampered by the relatively high background titres observed in the mock-immunized adjuvant control groups which may demonstrate increased unspecific reaction in the directly coated ELISA. The increased titres in the mock controls were observed mainly with the Pfs $48 / 45$ and AnAPN1 groups and to much less extent in the OVA group (Figures 1, 2, and 3). The Western Blot controls performed in parallel only displayed a specific but weak reaction in the Pfs48/45 groups immunized in conjunction with OMV or MF59C.1 (Figure 2). Such delayed isotype switch has been described upon infection [47]. Cellular responses against Pfs $48 / 45$ could not be detected due to toxicity of the protein when used in the restimulation protocol (Figure S4). Pfs $48 / 45$ cytotoxicity in vivo, however, seems not to be relevant. The detected antibody response requires CD4 T helper cell support and thus proves the existence and function of these $\mathrm{T}$ cells in vaccinated mice [48]. Furthermore, no significant side effects or reduced weight gain was observed in the experimental groups confronted with Pfs $48 / 45$ compared to the other antigens and the application controls arguing for negligible toxic effects on the mouse in vivo.

\section{Conclusions}

The results of this study argue that, based on the antigenadjuvant cocktail chosen, humoral and cellular immune responses may be shaped differently. They also suggest that i.n. vaccination with OMV may be a potent strategy to enhance intranasal heterologous vaccine antigens. OMVevoked antibody titres and T cell responses were about as strong as after i.n. immunization with CT. This aspect is of special importance as OMV application to the nasal mucosa may eventually be considered an easy and safe vaccination route as compared to CT, which is not used in humans due to toxicity. Importantly, i.n. vaccination led to robust and reproducible immune responses, indicating that antigen application and uptake do not limit the approach, even using antigens of large size and with strong charge such as Pfs $48 / 45$. Parenteral applications such as the MF59C.1 adjuvated group used here provide an almost complete antigen bioavailability; however, the immune responses were not found to be significantly better than with mucosal routes with uncertain bioavailability. The results provide the rationale for further improvement and engineering of OMV to facilitate their adherence to mucosal surfaces and boost of the immune response. Further studies are also aiming at directly coupling (malaria) antigens to OMV and at generating transgenic bacterial strains where supernatant can be used directly as a vaccine for use in resource limited settings.

\section{Disclosure}

The funders had no role in study design, data collection and analysis, decision to publish, or preparation of the paper.

\section{Competing Interests}

The authors declare that they have no competing interests.

\section{Authors' Contributions}

Michael Pritsch, Sebastian Kobold, Michael Hoelscher, and Andreas Wieser designed the experiments. Michael Pritsch, Najib Ben-Khaled, Michael Chaloupka, Annabell Peter, Gabriele Liegl, and Andreas Wieser performed laboratory work. Michael Pritsch, Najib Ben-Khaled, Sebastian Kobold, Nicole Berens-Riha, and Andreas Wieser undertook data analysis. Michael Pritsch, Sebastian Kobold, and Andreas Wieser wrote the first draft of the paper. All authors participated in interpretation as well as revision of the paper and approved the final version for submission.

\section{Acknowledgments}

This study was supported by Clinical Leave Scholarships from the German Center for Infection Research (to Michael Pritsch and Andreas Wieser). Further support was given from the Max von Pettenkofer Institute (to Andreas Wieser and Sören Schubert). This work was also supported by the international doctoral program " $\mathrm{i}$-Target: Immunotargeting of Cancer" funded by the Elite Network of Bavaria (to Sebastian Kobold), the Melanoma Research Alliance (Grant no. N269626 to Sebastian Kobold), the Wilhelm Sander Stiftung (Grant no. 2014.018.1 to Sebastian Kobold), the Graduiertenkolleg 1202 "Oligonucleotides in Cell Biology and Therapy" funded by the Deutsche Forschungsgemeinschaft (to Sebastian Kobold), the German Cancer Aid (to Sebastian Kobold), and the Else-Kröner-Fresenius Stiftung (to Sebastian Kobold).

\section{References}

[1] F. E. Andre, R. Booy, H. L. Bock et al., "Vaccination greatly reduces disease, disability, death and inequity worldwide," Bulletin of the World Health Organization, vol. 86, no. 2, pp. 140146, 2008. 
[2] S. L. Plotkin and S. A. Plotkin, "A short history of vaccination," in Vaccines, S. L. Plotkin, S. A. Plotkin, and P. A. Offit, Eds., pp. 1-13, Elsevier Saunders, Philadelphia, Pa, USA, 6th edition, 2013.

[3] C. A. Siegrist, "Vaccine immunology," in Vaccines, S. L. Plotkin, S. A. Plotkin, and P. A. Offit, Eds., pp. 14-32, Elsevier Saunders, Philadelphia, Pa, USA, 6th edition, 2013.

[4] N. Garçon, S. Hem, and M. Friede, "Evolution of adjuvants across the centuries," in Vaccines, S. L. Plotkin, S. A. Plotkin, and P. A. Offit, Eds., pp. 58-70, Elsevier Saunders, Philadelphia, Pa, USA, 6th edition, 2013.

[5] J. Holmgren and C. Czerkinsky, "Mucosal immunity and vaccines," Nature Medicine, vol. 11, no. 4, supplement, pp. S45-S53, 2005.

[6] B. G. Weniger and M. J. Papania, "Alternative vaccine delivery methods," in Vaccines, S. L. Plotkin, S. A. Plotkin, and P. A. Offit, Eds., pp. 1200-1231, Elsevier Saunders, Philadelphia, Pa, USA, 6th edition, 2013.

[7] H. Petersen, W. Nieves, K. Russell-Lodrigue, C. J. Roy, and L. A. Morici, "Evaluation of a Burkholderia pseudomallei outer membrane vesicle vaccine in nonhuman primates," Procedia in Vaccinology, vol. 8, pp. 38-42, 2014.

[8] K. S. Choi, S.-H. Kim, E.-D. Kim et al., "Protection from hemolytic uremic syndrome by eyedrop vaccination with modified enterohemorrhagic E. coli outer membrane vesicles," PLoS ONE, vol. 9, no. 7, Article ID e100229, 2014.

[9] J. A. Rosenthal, C. Huang, A. M. Doody et al., "Mechanistic insight into the $\mathrm{T}_{\mathrm{H}}{ }^{1}$-biased immune response to recombinant subunit vaccines delivered by probiotic bacteria-derived outer membrane vesicles," PLoS ONE, vol. 9, no. 11, Article ID el12802, 2014.

[10] B. Haneberg, R. Dalseg, E. Wedege et al., "Intranasal administration of a meningococcal outer membrane vesicle vaccine induces persistent local mucosal antibodies and serum antibodies with strong bactericidal activity in humans," Infection and Immunity, vol. 66, no. 4, pp. 1334-1341, 1998.

[11] B. Haneberg, R. Dalseg, F. Oftung et al., "Towards a nasal vaccine against meningococcal disease, and prospects for its use as a mucosal adjuvant," Developments in Biological Standardization, vol. 92, pp. 127-133, 1992.

[12] G. Sardiñas, K. Reddin, R. Pajon, and A. Gorringe, "Outer membrane vesicles of Neisseria lactamica as a potential mucosal adjuvant," Vaccine, vol. 24, no. 2, pp. 206-214, 2006.

[13] WHO-World Health Organization, World Malaria Report, 2014, http://www.who.int/malaria/publications/world_malaria _report_2014/en/.

[14] C. J. L. Murray, L. C. Rosenfeld, S. S. Lim et al., "Global malaria mortality between 1980 and 2010: a systematic analysis," The Lancet, vol. 379, no. 9814, pp. 413-431, 2012.

[15] R. R. Dinglasan, D. E. Kalume, S. M. Kanzok et al., "Disruption of Plasmodium falciparum development by antibodies against a conserved mosquito midgut antigen," Proceedings of the National Academy of Sciences of the United States of America, vol. 104, no. 33, pp. 13461-13466, 2007.

[16] R. R. Dinglasan, J. S. Armistead, J. F. Nyland, X. Jiang, and H. Q. Mao, "Single-dose microparticle delivery of a malaria transmission-blocking vaccine elicits a long-lasting functional antibody response," Current Molecular Medicine, vol. 13, no. 4, pp. 479487, 2013.
[17] J. S. Armistead, I. Morlais, D. K. Mathias et al., "Antibodies to a single, conserved epitope in anopheles APN1 inhibit universal transmission of Plasmodium falciparum and Plasmodium vivax malaria," Infection and Immunity, vol. 82, no. 2, pp. 818-829, 2014.

[18] D. K. Mathias, J. L. Plieskatt, J. S. Armistead et al., "Expression, immunogenicity, histopathology, and potency of a mosquitobased malaria transmission-blocking recombinant vaccine," Infection and Immunity, vol. 80, no. 4, pp. 1606-1614, 2012.

[19] R. L. B. Milek, W. F. G. Roeffen, C. H. M. Kocken et al., "Immunological properties of recombinant proteins of the transmission blocking vaccine candidate, Pfs $48 / 45$, of the human malaria parasite Plasmodium falciparum produced in Escherichia coli," Parasite Immunology, vol. 20, no. 8, pp. 377-385, 1998.

[20] B. Mulder, T. Lensen, T. Tchuinkam et al., "Plasmodium falciparum: membrane feeding assays and competition ELISAs for the measurement of transmission reduction in sera from Cameroon," Experimental Parasitology, vol. 92, no. 1, pp. 81-86, 1999.

[21] W. Roeffen, K. Teelen, J. Van As, M. Vd Vegte-Bolmer, W. Eling, and R. Sauerwein, "Plasmodium falciparum: production and characterization of rat monoclonal antibodies specific for the sexual-stage Pfs48/45 antigen," Experimental Parasitology, vol. 97, no. 1, pp. 45-49, 2001.

[22] W. F. G. Roeffen, J. M. H. Raats, K. Teelen et al., "Recombinant human antibodies specific for the Pfs $48 / 45$ protein of the malaria parasite Plasmodium falciparum," The Journal of Biological Chemistry, vol. 276, no. 23, pp. 19807-19811, 2001.

[23] N. S. Outchkourov, W. Roeffen, A. Kaan et al., "Correctly folded Pfs48/45 protein of Plasmodium falciparum elicits malaria transmission-blocking immunity in mice," Proceedings of the National Academy of Sciences of the United States of America, vol. 105, no. 11, pp. 4301-4305, 2008.

[24] N. Outchkourov, A. Vermunt, J. Jansen et al., "Epitope analysis of the malaria surface antigen pfs48/45 identifies a subdomain that elicits transmission blocking antibodies," The Journal of Biological Chemistry, vol. 282, pp. 17148-17156, 2007.

[25] A. E. Barry, L. Schultz, C. O. Buckee, and J. C. Reeder, "Contrasting population structures of the genes encoding ten leading vaccine-candidate antigens of the human malaria parasite, Plasmodium falciparum," PLoS ONE, vol. 4, no. 12, Article ID e8497, 2009.

[26] D. R. Chowdhury, E. Angov, T. Kariuki, and N. Kumar, "A potent malaria transmission blocking vaccine based on codon harmonized full length Pfs48/45 expressed in Escherichia coli," PLoS ONE, vol. 4, no. 7, Article ID e6352, 2009.

[27] M. Theisen, W. Roeffen, S. K. Singh et al., "A multi-stage malaria vaccine candidate targeting both transmission and asexual parasite life-cycle stages," Vaccine, vol. 32, no. 22, pp. 2623-2630, 2014.

[28] A. Wieser, E. Romann, G. Magistro et al., "A multiepitope subunit vaccine conveys protection against extraintestinal pathogenic Escherichia coli in mice," Infection and Immunity, vol. 78, no. 8, pp. 3432-3442, 2010.

[29] A. Wieser, E. Storz, G. Liegl et al., "Efficient quantification and characterization of bacterial outer membrane derived nanoparticles with flow cytometric analysis," International Journal of Medical Microbiology, vol. 304, no. 8, pp. 1032-1037, 2014. 
[30] J.-P. M. Valensi, J. R. Carlson, and G. A. Van Nest, "Systemic cytokine profiles in BALB/c mice immunized with trivalent influenza vaccine containing MF59 oil emulsion and other advanced adjuvants," The Journal of Immunology, vol. 153, no. 9, pp. 4029-4039, 1994.

[31] D. J. Chen, N. Osterrieder, S. M. Metzger et al., "Delivery of foreign antigens by engineered outer membrane vesicle vaccines," Proceedings of the National Academy of Sciences of the United States of America, vol. 107, no. 7, pp. 3099-3104, 2010.

[32] P. Mascagni, E. Vicenzi, A. Kajaste-Rudnitski et al., "Assessment of efficacy and safety of pandemic A/H1N1/2009 influenza vaccine in a group of health care workers," Medicina del Lavoro, vol. 103, no. 3, pp. 220-229, 2012.

[33] M. E. Armstrong, E. C. Lavelle, C. E. Loscher, M. A. Lynch, and K. H. G. Mills, "Proinflammatory responses in the murine brain after intranasal delivery of cholera toxin: implications for the use of $\mathrm{AB}$ toxins as adjuvants in intranasal vaccines," Journal of Infectious Diseases, vol. 192, no. 9, pp. 1628-1633, 2005.

[34] B. S. Collins, "Gram-negative outer membrane vesicles in vaccine development," Discovery Medicine, vol. 12, no. 62, pp. 7-15, 2011.

[35] J. C. Marshall, "Lipopolysaccharide: an endotoxin or an exogenous hormone?" Clinical Infectious Diseases, vol. 41, supplement 7, pp. S470-S480, 2005.

[36] B. Maletto, A. Rópolo, V. Morón, and M. C. Pistoresi-Palencia, "CpG-DNA stimulates cellular and humoral immunity and promotes Th1 differentiation in aged BALB/c mice," Journal of Leukocyte Biology, vol. 72, no. 3, pp. 447-454, 2002.

[37] J. Vadolas, J. K. Davies, P. J. Wright, and R. A. Strugnell, "Intranasal immunization with liposomes induces strong mucosal immune responses in mice," European Journal of Immunology, vol. 25, no. 4, pp. 969-975, 1995.

[38] J. W. Huleatt, A. R. Jacobs, J. Tang et al., "Vaccination with recombinant fusion proteins incorporating Toll-like receptor ligands induces rapid cellular and humoral immunity," Vaccine, vol. 25, no. 4, pp. 763-775, 2007.

[39] C. M. Snapper and W. E. Paul, "Interferon- $\gamma$ and B cell stimulatory factor-1 reciprocally regulate Ig isotype production," Science, vol. 236, no. 4804, pp. 944-947, 1987.

[40] L. M. Næss, E. Rosenqvista, E. A. Høiby, and T. E. Michaelsen, "Quantitation of IgG subclass antibody responses after immunization with a group B meningococcal outer membrane vesicle vaccine, using monoclonal mouse-human chimeric antibodies as standards," Journal of Immunological Methods, vol. 196, no. 1, pp. 41-49, 1996.

[41] H. T. Maecker, D. T. Umetsu, R. H. DeKruyff, and S. Levy, "Cytotoxic T cell responses to DNA vaccination: dependence on antigen presentation via class II MHC," The Journal of Immunology, vol. 161, no. 12, pp. 6532-6536, 1998.

[42] M. J. Estcourt, A. J. McMichael, and T. Hanke, "Altered primary $\mathrm{CD}^{+} \mathrm{T}$ cell response to a modified virus Ankara(MVA)vectored vaccine in the absence of $\mathrm{CD} 4^{+} \mathrm{T}$ cell help," European Journal of Immunology, vol. 35, no. 12, pp. 3460-3467, 2005.

[43] J. Ménager, F. Ebstein, R. Oger et al., "Cross-presentation of synthetic long peptides by human dendritic cells: a process dependent on ERAD component p97/VCP but not sec61 and/or Derlin-1," PLoS ONE, vol. 9, no. 2, Article ID e89897, 2014.

[44] J. Bayry, "Regulatory T cells as adjuvant target for enhancing the viral disease vaccine efficacy," VirusDisease, vol. 25, no. 1, pp. 18$25,2014$.
[45] K. J. Ewer, G. A. O’Hara, C. J. A. Duncan et al., "Protective CD8 ${ }^{+}$ T-cell immunity to human malaria induced by chimpanzee adenovirus-MVA immunisation," Nature Communications, vol. 4, article 2836, 2013.

[46] B. J. McMahon, C. M. Dentinger, D. Bruden et al., "Antibody levels and protection after hepatitis b vaccine: results of a 22 year follow-up study and response to a booster dose," Journal of Infectious Diseases, vol. 200, no. 9, pp. 1390-1396, 2009.

[47] T. F. Marandu, K. Finsterbusch, A. Kröger, and L. Čičin-Šain, "Mouse CMV infection delays antibody class switch upon an unrelated virus challenge," Experimental Gerontology, vol. 54, pp. 101-108, 2014.

[48] C. A. Janeway, P. Travers, M. Walport, and M. J. Shlomchik, Eds., Immunobiology, vol. 5th, Garland Science, New York, NY, USA, 2001. 


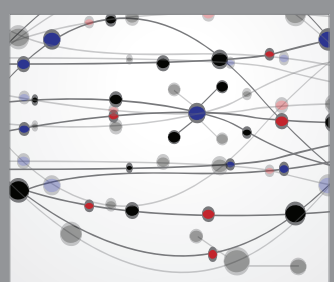

The Scientific World Journal
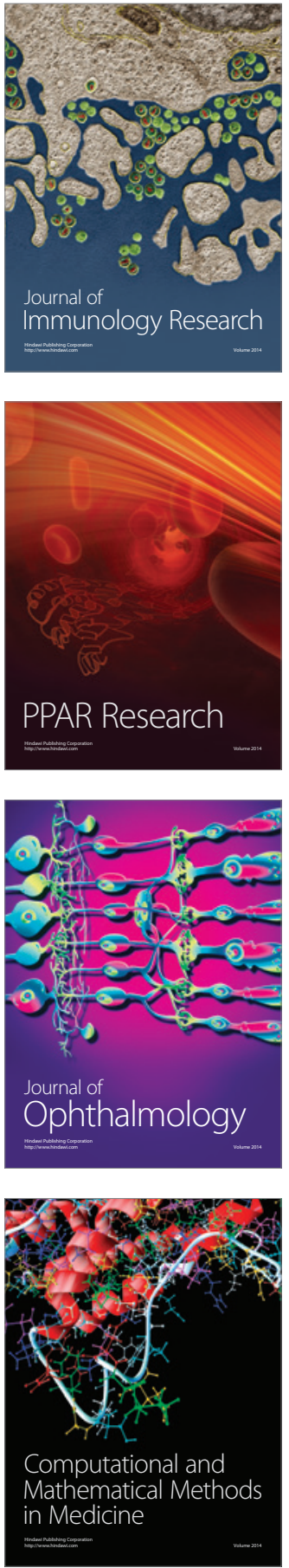

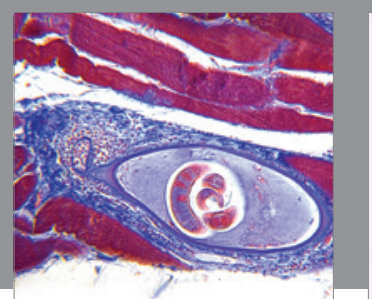

Gastroenterology Research and Practice

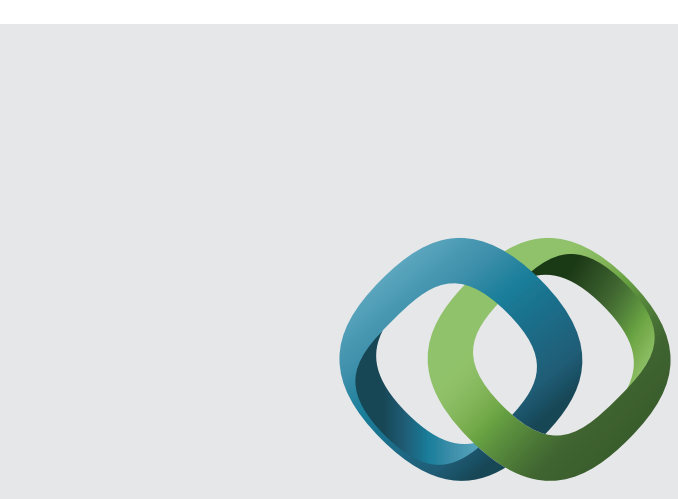

\section{Hindawi}

Submit your manuscripts at

http://www.hindawi.com
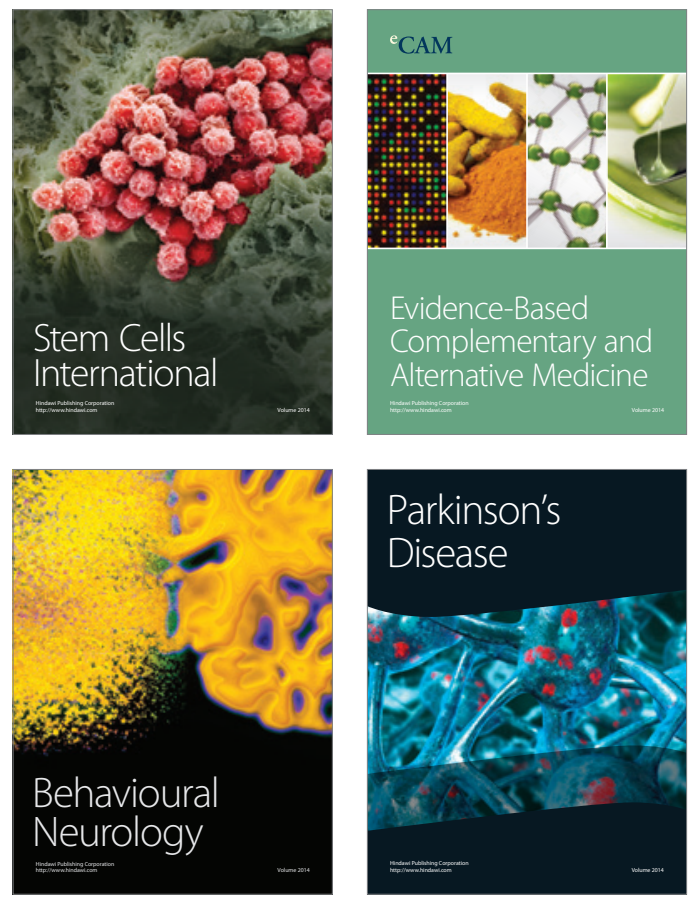
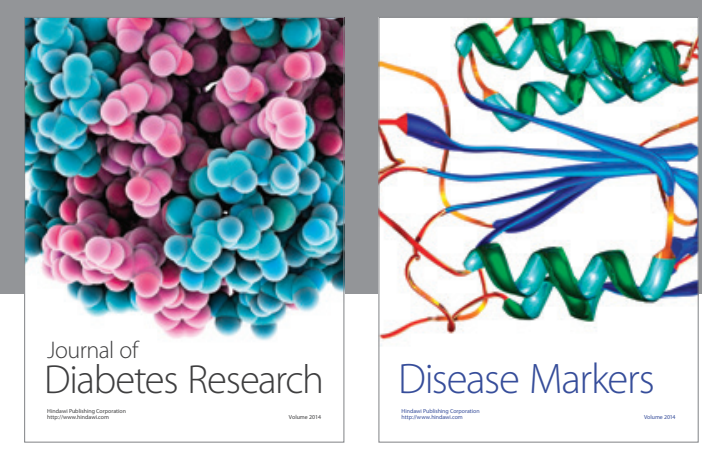

Disease Markers
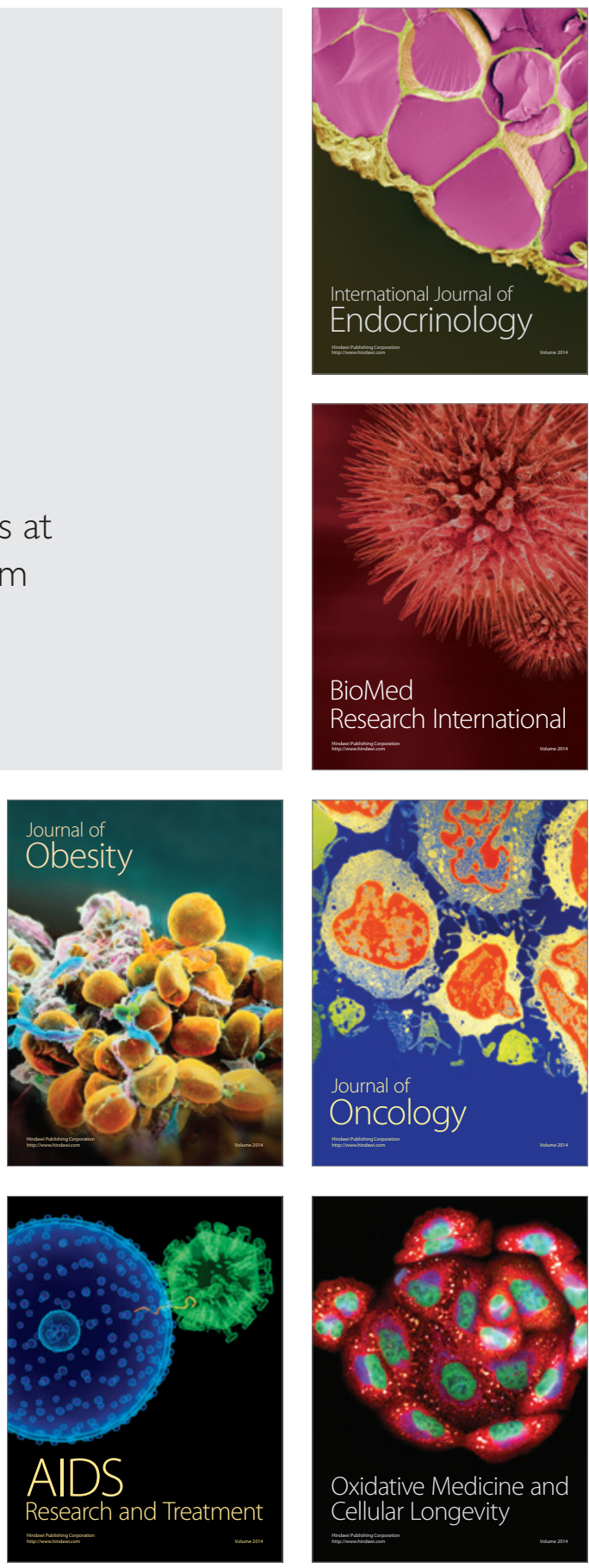